\title{
Skeletal muscle satellite cell-derived mesenchymal stem cells ameliorate acute alcohol-induced liver injury
}

Jae Sik Chung ${ }^{*}$, Soonjae Hwang2,3*, Ju Eun Hong ${ }^{4}$, Minjeong Jo ${ }^{4}$, Ki-Jong Rhee ${ }^{4}$, Seongyup Kim ${ }^{1}$, Pil Young Jung1, Youngdae Yoon ${ }^{2,5}$, Seong Hee Kang 2,6 , Hoon Ryu ${ }^{1}$, Moon Young Kim²,6,7凶 , Keum Seok Bae ${ }^{\bowtie}$ and Young Woo Eom $2,7 \bowtie$

1. Department of Surgery, Yonsei University Wonju College of Medicine, Wonju, Gangwon-do 26426, Republic of Korea.

2. Regeneration Medicine Research Center, Yonsei University Wonju College of Medicine, Wonju, Gangwon-do 26426, Republic of Korea.

3. Department of Biochemistry, Lee Gil Ya Cancer and Diabetes Institute, GAIHST, Gachon University College of Medicine, Incheon 21999, Republic of Korea.

4. Department of Biomedical Laboratory Science, College of Health Sciences, Yonsei University MIRAE Campus, Wonju, Gangwon-do 26493, Republic of Korea

5. Mitohormesis Research Center, Yonsei University Wonju College of Medicine, Wonju, Gangwon-do 26426, Republic of Korea.

6. Department of Internal Medicine, Yonsei University Wonju College of Medicine, Wonju, Gangwon-do 26426, Republic of Korea.

7. Cell Therapy and Tissue Engineering Center, Yonsei University Wonju College of Medicine, Wonju, Gangwon-do 26426, Republic of Korea.

*These authors contributed equally to this work.

$\triangle$ Corresponding authors: Moon Young Kim, Department of Internal Medicine, Yonsei University Wonju College of Medicine, Wonju, Gangwon-do 26426, Republic of Korea. E-mail: drkimmy@yonsei.ac.kr; Keum Seok Bae, Department of General Surgery, Yonsei University Wonju College of Medicine, Wonju, Gangwon-do 26426, Republic of Korea. Tel: + 82-33-741-0573. E-mail: bksgs@yonsei.ac.kr; Young Woo Eom, Cell Therapy and Tissue Engineering Center and Regeneration Medicine Research Center, Yonsei University Wonju College of Medicine, Wonju, Gangwon-do 26426, Republic of Korea. E-mail: yweom@yonsei.ac.kr.

(C) The author(s). This is an open access article distributed under the terms of the Creative Commons Attribution License (https://creativecommons.org/licenses/by/4.0/). See http://ivyspring.com/terms for full terms and conditions.

Received: 2021.11.11; Accepted: 2022.01.05; Published: 2022.01.24

\begin{abstract}
Cultured human skeletal-muscle satellite cells have properties of mesenchymal stem cells (skeletal muscle satellite cell-derived mesenchymal stem cells, SkMSCs) and play anti-inflammatory roles by secreting prostaglandin E2 and hepatocyte growth factor (HGF). To evaluate the utility of SkMSCs in treating liver diseases, we determined whether SkMSCs could ameliorate acute liver and gut inflammation induced by binge ethanol administration. Binge drinking of ethanol led to weight loss in the body and spleen, liver inflammation and steatosis, and increased serum ALT and AST levels (markers of liver injury), along with increased IL-1 $\beta$, TNF- $\alpha$, and iNOS expression levels in mice. However, levels of these binge-drinking-induced indicators were reduced by a single intraperitoneal treatment of SkMSCs. Furthermore, levels of bacteria-derived lipopolysaccharide decreased in the livers and sera of ethanol-exposed mice after SkMSC administration. SkMSCs decreased the extent of tissue inflammation and reduced villus and crypt lengths in the small intestine after alcohol binge drinking. SkMSCs also reduced the leakage of blood albumin, an indicator of leaky gut, in the stool of ethanol-exposed mice. Alcohol-induced damage to human colonic Caco-2/tc7 cells was also alleviated by HGF. Therefore, a single treatment with SkMSCs can attenuate alcoholic liver damage by reducing inflammatory responses in the liver and gut, suggesting that SkMSCs could be used in cell therapy to treat alcoholic liver diseases.
\end{abstract}

Key words: skeletal muscle satellite cells; mesenchymal stem cells; binge ethanol; inflammation; liver; gut

\section{Introduction}

Alcoholic liver disease can progress from simple hepatic steatosis and steatohepatitis to end-stage organ failure (cirrhosis). Cirrhosis is the tenth leading cause of mortality in developed countries [1]. Alcohol consumption is a major cause of this disease approximately $50 \%$ of all cirrhosis-associated mortalities are associated with alcohol consumption $[2,3]$. Chronic alcohol abusers and patients with alcoholic liver disease exhibit increased serum endotoxin levels and a disrupted gut barrier with increased permeability [4-6]. Alcohol-associated liver inflammation is mediated by commensal gut microbederived endotoxins, which induce TLR4-mediated inflammatory signaling as well as hepatic steatosis in mice [7-9]. Such findings from animal models and alcoholic patients indicate that alcohol-induced liver injury is associated with inflammation of the gut barrier (the intestinal epithelium) that maintains the immunologic homeostasis between the gut tissue and resident microbiota. Alcohol-associated hepatic 
steatosis and steatohepatitis result from enteric bacteria as germ-free mice display reduced extent of liver inflammation and alcohol-fed mice administered with antibiotics display decreased levels of hepatic steatosis [10-12].

Curative treatment for alcohol-promoted cirrhosis is currently limited to orthotropic liver transplantation. However, a global shortage of donor organs has resulted in the death of patients on the organ waitlist [13]. Therefore, stem cell-based therapy has emerged as a promising alternative with accumulating evidence of its efficacy in experimental and clinical studies [14-17]. Adult stem cells have been isolated from the bone marrow and identified as mesenchymal stem cells (MSCs) [18]. The self-renewal and differentiation abilities of these cells are of great interest in cell-based therapies [17]. Alternate sources of MSCs, such as umbilical cord tissue, blood, liver, dental pulp, and skin, have been investigated; the functions of MSCs depend on the collection procedure, cell quantity, immaturity, and cell profile $[19,20]$. MSCs originating from different sources are known to play a role in healing and repairing damaged tissues through secreted cytokines [21].

Although skeletal-muscle satellite cells (SCs) are important effector cells for the regeneration of damaged muscle, other resident cell types, including motor neurons, endothelial cells, immune cells, and fibro-adipogenic progenitors (FAPs), play a positive role in muscle repair and homeostasis [22-24]. The acute inflammatory response after muscle injury induces the infiltration of neutrophils and M1 macrophages [5,6], leading to SC activation [7] and extracellular matrix production by FAPs. FAPs induce the regeneration of damaged muscle tissue and maintain homeostasis in muscle tissue [22, 25]. According to a previous study by our group, cultured SCs showed MSC-like phenotypes (skeletal muscle satellite cell-derived mesenchymal stem cells, SkMSCs), including anti-inflammatory effects on macrophages through PGE2 and HGF, leading to the inhibition of IL-1 $\beta$ secretion [26]. In addition, the population doubling time of SkMSCs is markedly shorter than that of MSCs isolated from bone marrow or adipose tissue, which is expected to be a great advantage for cell therapy using adult stem cells [26]. Endotoxins derived from intestinal microorganisms have been reported to migrate to liver tissue owing to excessive alcohol intake and induce damage in small intestinal epithelial cells, promoting alcoholassociated hepatic steatosis and steatohepatitis through the activation of immune cells, including neutrophils and macrophages, which exacerbates liver injury [27, 28]. However, whether adult stem cells can reduce alcohol-induced hepatic and intestinal damage in a mouse model of alcoholinduced liver damage has not been investigated. In this study, we aimed to determine whether SkMSCs could treat alcohol-induced inflammatory liver injury and gut inflammation in mice.

\section{Materials and Methods}

\section{SkMSC cell culture}

This study was approved by the Institutional Review Board of Yonsei University Wonju College of Medicine (CR320308). Human skeletal muscle satellite cells (SCs) at passage (P) 1 were purchased from ScienCell (Cat no. 3510, Carlsbad, CA, USA), seeded in $100-\mathrm{mm}$ dishes coated with poly-L-lysine (ScienCell), and subcultured in skeletal muscle cell medium (SkMCM, ScienCell) supplemented with 2\% fetal bovine serum, antibiotics, and skeletal muscle cell growth supplement (SkMCGS, ScienCell). Upon confirmation at $\mathrm{P} 5$, the isolated cells showed characteristics of MSCs rather than those of satellite cells [26]. Accordingly, P5 cells were used for this experiment.

\section{Animal treatments}

All animal housing and experimental procedures were reviewed and approved by the Institutional Animal Care and Use Committee of Yonsei University at Wonju (YWCI-201909-013-01 and YWC-200305-1). All mice were maintained under controlled lighting (12-h light/dark cycle) with food and water provided ad libitum. Age-matched 8-10-week-old female C57BL/ 6 wild-type (WT) mice were administered a single intraperitoneal (i.p.) injection of $2.0 \times 10^{6}$ SkMSCs $/ 200 \mu \mathrm{L}$ in Hanks' balanced salt solution (HBSS, Sigma, San Diego, CA, USA) on day 2 after receiving a second binge alcohol dose $(6 \mathrm{~g} / \mathrm{kg} /$ dose $)$. Control mice were administered a vehicle (phosphatebuffered saline [PBS], Gibco BRL, Rockville, MD, USA). Mice were administered three oral doses of binge alcohol (6 g/ kg/dose) or dextrose (as control) at 12-h intervals and euthanized at $1 \mathrm{~h}$ after the last ethanol treatment.

\section{Histology}

The liver and small intestine (ileum) were excised and fixed in 10\% formalin. For microscopic examination, tissues were embedded in paraffin and sectioned $(4 \mu \mathrm{m})$ using a rotary microtome. Slides were stained with hematoxylin, bluing buffer, and eosin for 1 min each; dehydrated using alcohol (95\%, $100 \%, 100 \%$, and $100 \%$ ) for $1 \mathrm{~min}$ each; and rinsed twice with xylene for $1 \mathrm{~min}$ each. Slides were photographed using an optical microscope (Leica, Wetzlar, Germany) and rendered using Adobe Photoshop and Leica software. Histological scoring of 
the liver was performed based on lobular inflammation and steatosis. Lobular inflammation scores of the liver during binge-induced liver damage were graded blindly by a board-certified pathologist as follows: 0 , none; 1, 1-2 foci $/ \times 20$ fields; $2,2-4$ foci/ $\times 20$ fields; and $3,>4$ foci $/ \times 20$ fields. Hepatic steatosis, characterized by hepatocytes containing fat vacuoles, was subjectively visualized by hematoxylin and eosin (H\&E) staining and graded according to the following scale: 0 , normal, no hepatocytes affected; 1 , minor, $<5 \%$ of hepatocytes affected; 2 , mild, $5 \%-33 \%$ of hepatocytes affected; 3, moderate, $34 \%-66 \%$ of hepatocytes affected; and 4, severe, $>66 \%$ of hepatocytes affected. Histological assessments of inflammation in the ileum were performed in a double-blind manner using H\&E-stained sections. Histological scoring of the ileum was performed based on the severity of inflammation, the extent of injury, regeneration, and crypt damage. The final inflammation score for the ileum was calculated as the sum of the scores for all parameters. Inflammation was evaluated as follows: 0 , normal; 1 , mild increase in immune cell numbers and no colonic epithelial alterations; 2, a moderate increase in immune cell numbers and mild epithelial proliferation; and 3, severe increase in immune cell numbers and aberrant epithelial proliferation with extensive loss of villi/crypt architecture. Villus and crypt lengths in the ileum were determined according to an analysis of 15 well-oriented villi/crypts per mouse using the image analysis software LAS 2.0 (Leica). The inflammation scores or villus and crypt lengths were calculated as the medians of individual measurements from the two colonic sections of each mouse.

\section{Measurements of endotoxin levels}

Serum endotoxin levels were analyzed using a commercially available endpoint LAL Chromogenic Endotoxin Quantitation Kit with a concentration range of 0.015-1.2 endotoxin units per milliliter (EU/mL; Thermo Fisher Scientific, Waltham, MA, USA), as previously described [29].

\section{Albumin enzyme-linked immunosorbent assay (ELISA)}

Mice were transferred to individual cages $1 \mathrm{~h}$ prior to sacrifice to collect mouse feces for blood albumin leakage analysis. At the beginning of the sacrifice, one fecal pellet per mouse was immediately collected into an autoclave tube using sterile forceps. If there was no stool in the cage, the last feces from the colon closest to the rectum was collected and used for further analysis. One fecal pellet was used for albumin analysis. Feces were dissolved in sample dilution buffer $(50 \mathrm{mM}$ Tris, $0.14 \mathrm{M} \mathrm{NaCl}, 0.05 \%$ tween20, $\mathrm{pH} 8.0$ ) to a concentration of $100 \mathrm{mg} / \mathrm{mL}$. Fecal albumin levels were quantified using a mouse albumin ELISA kit (Abcam, Cambridge, UK) according to the manufacturer's instructions. The results were subsequently quantified using a microplate reader (BioTek Instruments, Winooski, VT, USA). Human HGF was also analyzed using the HGF ELISA kit, and subsequently quantified using a microplate reader.

\section{Quantitative reverse-transcription polymerase chain reaction ( $q R T-P C R$ )}

Total RNA was isolated from individual mouse livers and the small intestine (ileum), with overnight precipitation at $-20{ }^{\circ} \mathrm{C}$ to increase the yield of RNA. Transcript levels were measured using the QuantStudio $^{\mathrm{TM}}$ Real-Time PCR System (Applied Biosystems, Waltham, MA, USA) and commercial TaqMan probes (Applied Biosystems). The following cycling condition was used for qRT-PCR: $95^{\circ} \mathrm{C}$ for 10 min, followed by 40 cycles of $95^{\circ} \mathrm{C}$ for $15 \mathrm{~s}, 55^{\circ} \mathrm{C}$ for $30 \mathrm{~s}$, and $72{ }^{\circ} \mathrm{C}$ for $1 \mathrm{~min}$. Data were normalized to GAPDH expression level, and the relative amount was calculated using the $2^{-\Delta \Delta C t}$ method.

\section{Liver enterobacteria assay}

To determine bacterial colonization in ethanolexposed mice, liver tissues were collected at the indicated time points after binge ethanol exposure. For the colony-forming unit (CFU) assays, liver contents were weighed, homogenized in sterile PBS, serially diluted, and seeded on sheep blood agar plates cultured overnight at $37{ }^{\circ} \mathrm{C}$ under anaerobic conditions (Pack-Anaero; Mitsubishi Gas Chemical, Tokyo, Japan).

\section{Nitric oxide assay}

Nitric oxide was measured as the amount of nitrite, which is the stable product of nitric oxide metabolism. Mouse sera were centrifuged at 4,000 rpm for $30 \mathrm{~min}$, and the supernatants were collected and measured by a colorimetric assay using the Griess reaction (Invitrogen, Carlsbad, CA, USA). Sera of individual mice $(100 \mu \mathrm{L})$ were incubated with an equal volume of Griess reagent at room temperature for $10 \mathrm{~min}$. After incubation, the absorbance of the wells was measured at $550 \mathrm{~nm}$ using a microplate reader (Tecan, Männedorf, Switzerland).

\section{Evaluation of Barrier Function in Caco-2/tc7 Cells}

Human colonic Caco-2/tc7 cells were maintained in Dulbecco's modified Eagle's medium (DMEM; Gibco, Rockville, MD, USA) containing 10\% fetal bovine serum (FBS; Gibco) and penicillin/streptomycin. Caco-2/tc7 cells were seeded 
on polycarbonate membrane Transwell inserts with a surface area of $0.33 \mathrm{~cm}^{2}(0.4 \mu \mathrm{m}$ pore size; SPL Life Science, Pocheon, Republic of Korea). After $1 \mathrm{~d}$ of cell seeding, the culture medium was replaced with fresh DMEM supplemented with $10 \%$ FBS and penicillin/streptomycin. The medium was changed three times throughout the week. After the trans-epithelial electrical resistance (TEER) exceeded $350 \Omega \mathrm{cm}^{2}$, intestinal barriers comprising Caco-2/tc7 cells were exposed to ethanol alone or co-treated with HGF for $24 \mathrm{~h}$. TEER was measured using a Millicell Electrical Resistance System (ERS) meter (Millipore Corporation, Bedford, MA, USA). Data were collected from quadruple inserts per treatment in three experiments and expressed as a percentage of basal TEER obtained before ethanol treatment. At the end of the TEER measurements, $50 \mu \mathrm{g} / \mu \mathrm{L}$ FITC labeled 4-kDa dextran (FITC-D4; Sigma-Aldrich, St. Louis, MO, USA) was added to the apical side of the upper chamber of transwell plates seeded with Caco-2/tc7 cells. After $1 \mathrm{~h}$ of FITC-D4 treatment, FITC-D4 permeability to intestinal barriers was analyzed by spectrophotometrically measuring the fluorescent signals in the culture media of bottom wells using a 96-well microplate reader (Molecular Devices, San Jose, CA, USA) at excitation and emission spectra of $485 \mathrm{~nm}$ and $540 \mathrm{~nm}$, respectively.

A
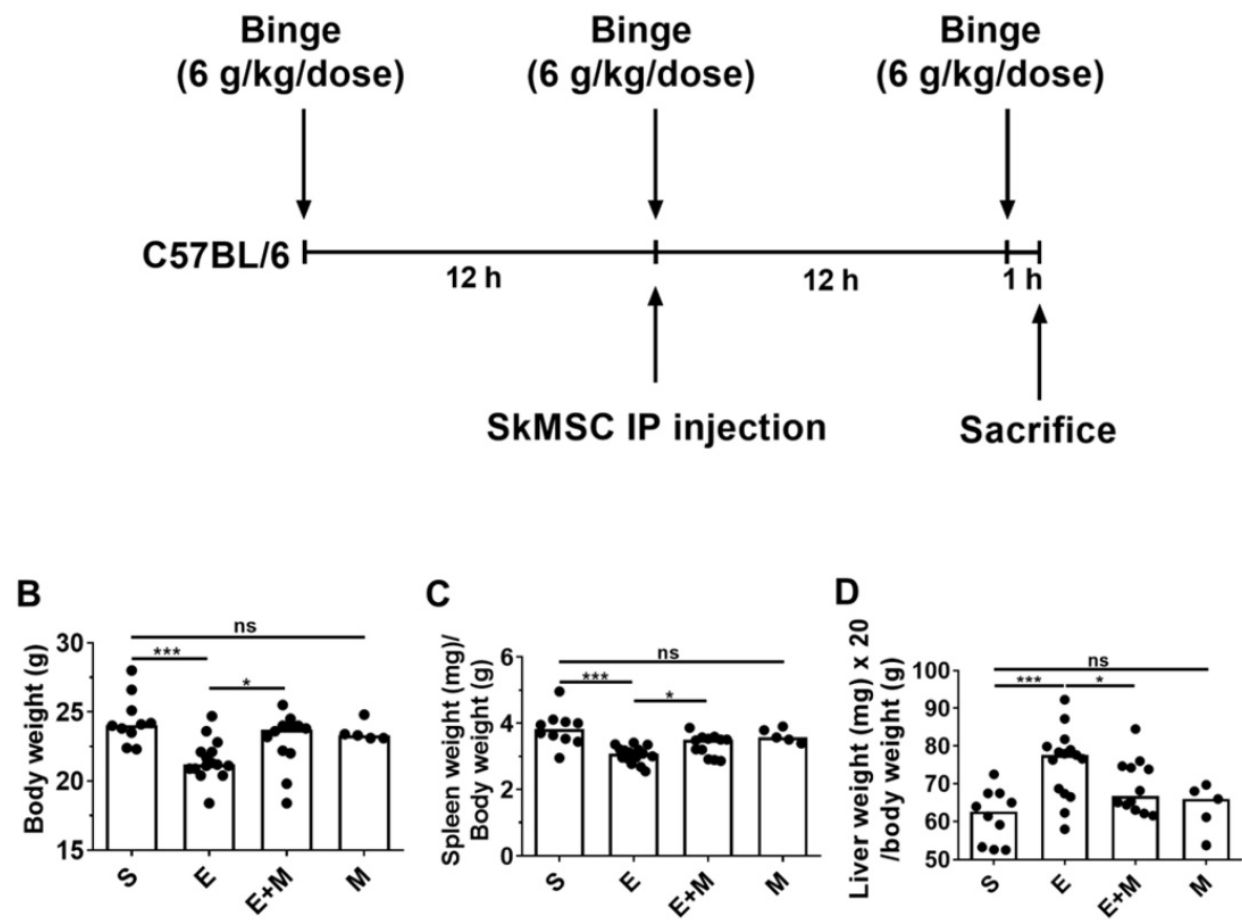

Figure 1. Experimental scheme and effects of skeletal muscle satellite cell-derived mesenchymal stem cells (SkMSCs) on body weight, spleen weight, and liver weight in binge ethanol-exposed mice. Eight-week-old C57BL/6 female mice were treated with binge ethanol and sacrificed on day 1. (A) Experimental design of the binge ethanol-induced liver injury model. (B-D) Body, spleen, and liver weight were measured after the third injection of ethanol. $\mathrm{n}=5-17$ mice per group. Scatter plot: horizontal bar, median. $\mathrm{S}$, sham; $\mathrm{E}$, binge ethanol; M, SkMSCs. Significance between treated groups was determined using Mann-Whitney $U$ test. $* P<0.05$, $* * * P<0.001$; ns, no statistical significance. 
A

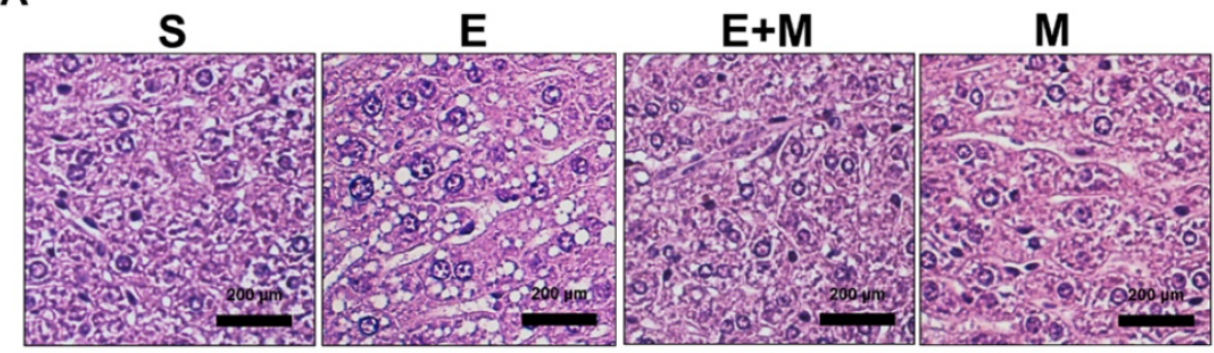

B

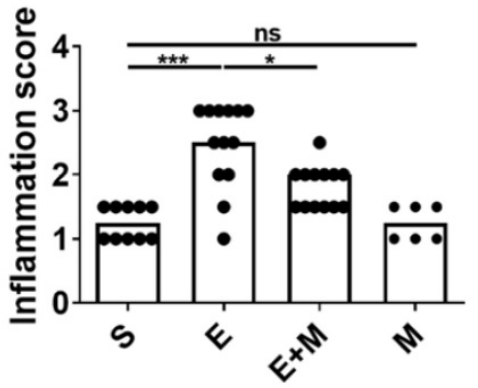

D

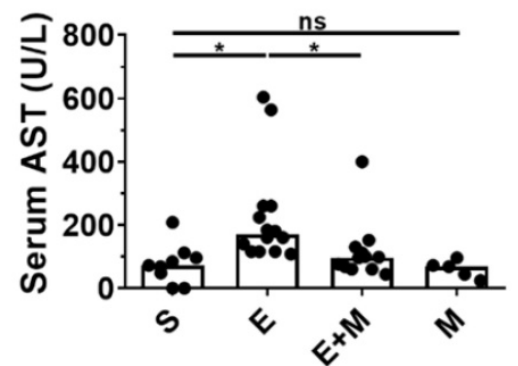

C

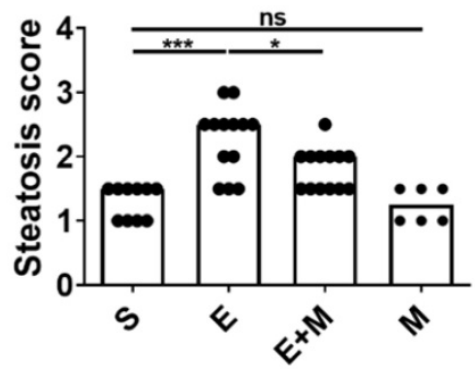

E

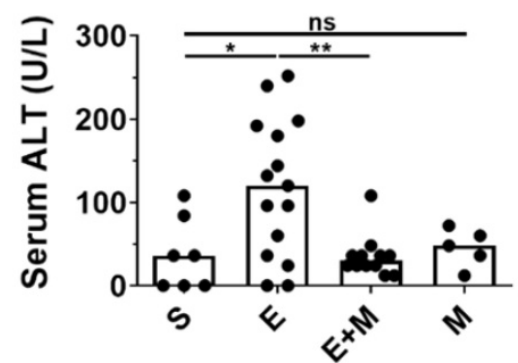

Figure 2. Effect of skeletal muscle satellite cell-derived mesenchymal stem cells (SkMSCs) on liver histology and serum AST and ALT levels in binge ethanol-treated mice. Eight-week-old C57BL/6 female mice were treated with binge ethanol. Mice were sacrificed on day 1 , and liver tissues were fixed and stained with H\&E. Representative images are shown. Histologic scores were evaluated using H\&E slides. Serum AST and ALT levels were analyzed by a colorimetric assay kit. (A) H\&E staining of the liver. (B) Inflammation score; (C) steatosis score; (D) serum AST levels; (E) serum ALT levels ( $n=5-17$ mice per group). Scatter plot: horizontal bar, median. S, sham; E, ethanol binge; M, SkMSCs. Significance between treated groups was determined using Mann-Whitney $U$ test. $* P<0.05$, *** $P<0.001$; ns, no statistical significance.

\section{Effect of SkMSCs on liver damage in binge ethanol-treated mice}

Oral administration of binge ethanol induces histological hepatic inflammation and steatosis [27, 28]. To determine whether treatment with SkMSCs could decrease ethanol-induced hepatic damage, mice were intraperitoneally administered SkMSCs, and hepatic inflammation and steatosis were histologically assessed at the end of the experimental period. Mice administered binge ethanol alone showed increased levels of inflammation and steatosis, as determined by H\&E staining, whereas mice treated with binge ethanol + SkMSCs showed decreased levels of inflammation and steatosis (Figure 2B, C). Histologically, the administration of SkMSCs alone did not induce hepatic inflammation and steatosis in mice (Figure 2A-C). To determine whether SkMSCs could decrease the induction of liver damage, hepatic tissues from ethanol-treated mice administered SkMSCs were analyzed for serum AST and ALT levels. Based on the results, binge ethanol-treated mice administered SkMSCs had a significant decrease in serum AST and ALT levels compared to ethanol-treated mice (Figure 2D, E). Mice administered SkMSCs alone had comparable serum AST and ALT levels to those in the sham group, which suggests that SkMSCs alone do not induce liver damage. Collectively, these data indicate that SkMSCs ameliorate ethanol-induced hepatic damage.

\section{Effect of SkMSCs on bacterial translocation and inflammatory mediators in the small intestine of binge ethanol-treated mice}

Disruption of the intestinal barrier caused by binge ethanol promotes the translocation of bacterial products, such as LPS, to the liver, where they interact with hepatic Toll-like receptors (TLRs) and stimulate liver inflammation and damage [27, 28, 34]. As SkMSCs decreased the generation of multiple lipid 
droplets in hepatocytes induced by binge ethanol (Figure 2A), as well as serum ALT and AST levels (Figure 2D, E), we hypothesized that they would decrease the translocation of LPS and intestinal bacteria, thereby suppressing inflammatory mediators, such as cytokines and reactive nitrogen species. To determine whether SkMSCs could decrease the translocation of bacterial products or gut microbes caused by ethanol binge drinking, serum and hepatic tissues of mice were analyzed for LPS and bacterial colonization using an endotoxin assay kit and anaerobic cultivation, respectively. The levels of LPS were found to be downregulated in ethanolexposed mice treated with SkMSCs compared to mice treated with binge ethanol alone (Figure 3A, B).

Consistently, the CFUs were reduced in ethanolexposed mice treated with SkMSCs compared to mice treated with binge ethanol alone (Figure 3A, B). Treatment with SkMSCs alone did not affect LPS levels in serum and CFUs in liver tissues, suggesting that they do not affect gut integrity. Additionally,

A

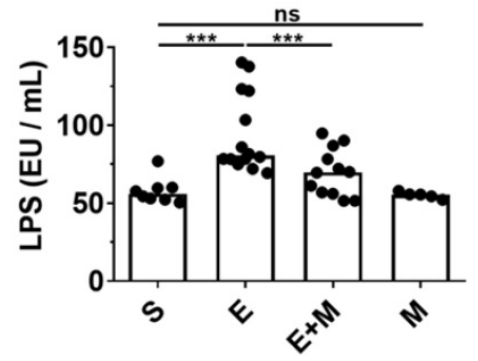

C

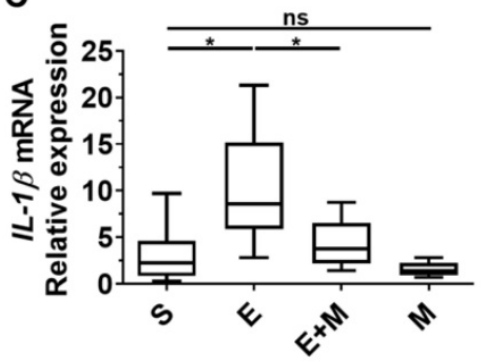

E

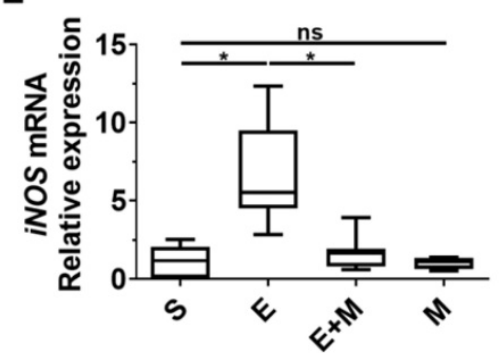

small intestinal tissues (ileum) from binge ethanol-exposed mice administered SkMSCs were analyzed for $I L-1 \beta$, TNF- $\alpha$, and $i N O S$ expression by qRT-PCR. Serum nitrite levels were also examined using a nitric oxide assay. Binge ethanol-exposed mice administered SkMSCs were found to display a decrease in $I L-1 \beta$, TNF- $\alpha$, and $i N O S$ expression levels compared to those in mice treated with ethanol alone (Figure 3C-E). Nitrite (a metabolite of nitric oxide) levels in the serum were also decreased in ethanolexposed mice administered SkMSCs, consistent with the iNOS expression data (Figure 3E), suggesting that SkMSCs decrease the binge ethanol-induced translocation of bacterial products and gut bacteria, simultaneously reducing the expression levels of inflammatory mediators. Collectively, these data support the hypothesis that SkMSC treatment decreases ethanol-induced hepatic damage and inflammation by suppressing the translocation of bacterial products and gut bacteria.

B

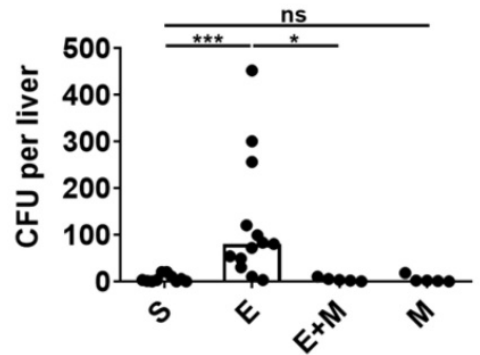

D

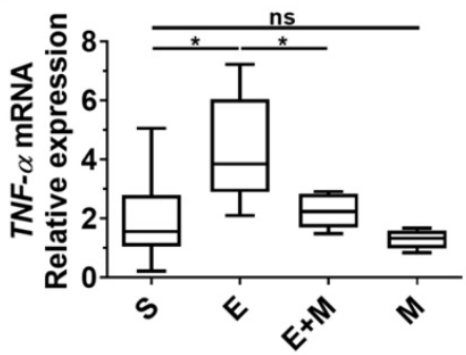

$\mathbf{F}$

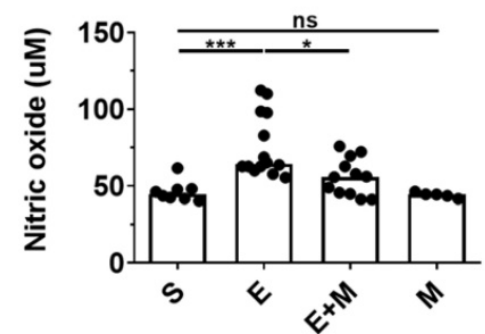

Figure 3. Effect of skeletal muscle satellite cell-derived mesenchymal stem cells (SkMSCs) on serum lipopolysaccharide (LPS), hepatic enterobacteria content, and pro-inflammatory genes in the liver tissues of binge ethanol-treated mice. Eight-week-old C57BL/6 female mice were treated with binge ethanol. Mice were sacrificed on day 1; serum and liver tissues were analyzed to determine LPS concentration using endotoxin activity assays and the mRNA expression levels of IL- $I \beta$, TNF- $\alpha$ and iNOS in the ileum were determined using qRT-PCR. Hepatic enterobacteria content in the liver tissues was analyzed using sheep blood agar plates. Sera of mice were analyzed to derive the nitrite content using nitric oxide assay kits. (A) Serum LPS levels; (B) average number of colonies in liver tissues; (C) IL-I $\beta$ expression levels in the ileum; (D) TNF- $\alpha$ expression levels in the ileum; (E) iNOS expression levels in the ileum; (F) serum nitric oxide levels ( $n=5-15$ mice per group). Scatter plot: horizontal bar, median. S, sham; $E$, ethanol binge; M, SkMSCs. Significance between treated groups was determined using Mann-Whitney $U$ test. $* P<0.05$, *** $P<0.001$; ns, no statistical significance. 
A

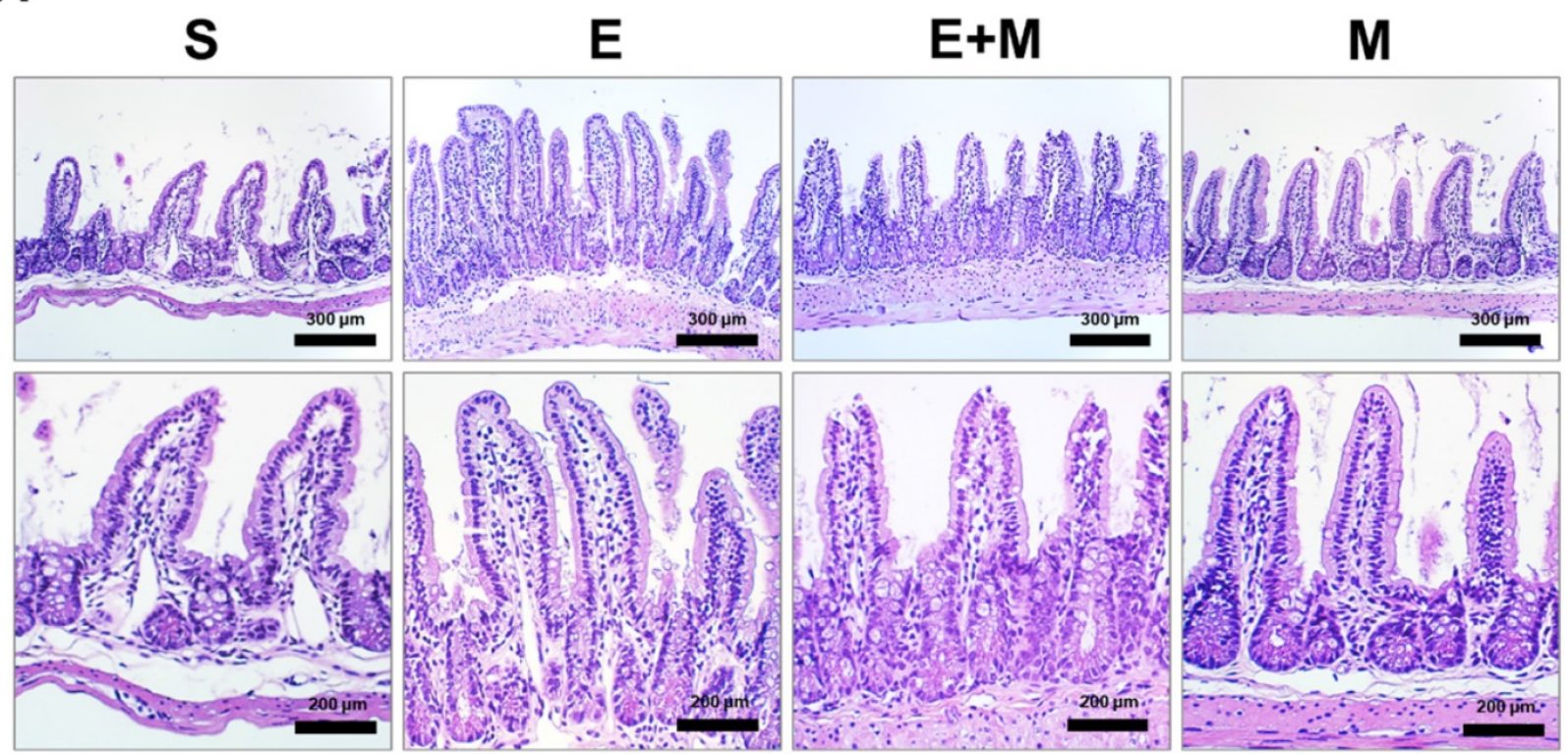

B

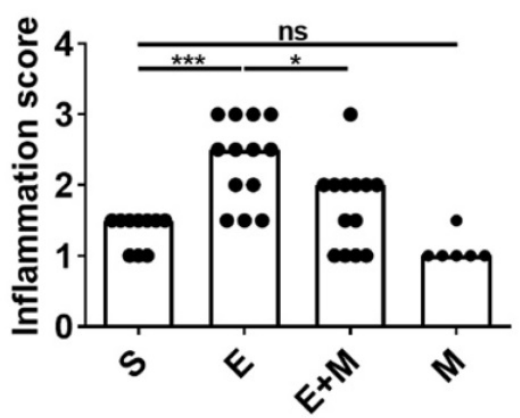

C

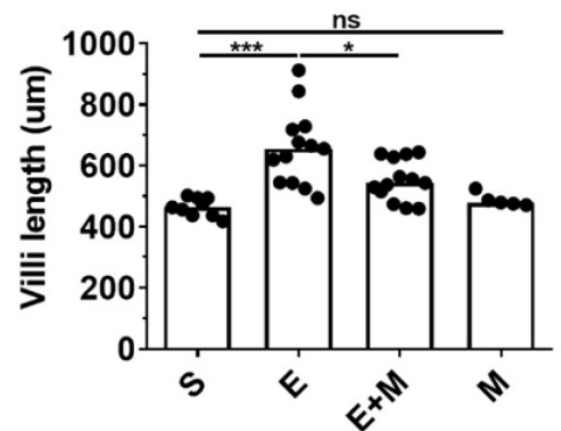

D

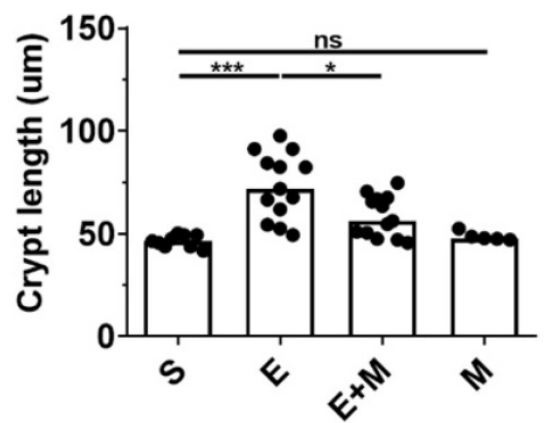

Figure 4. Effect of skeletal muscle satellite cell-derived mesenchymal stem cells (SkMSCs) on intestinal histopathology. Eight-week-old C57BL/6 female mice were treated with binge ethanol. Mice were sacrificed on day 1 , and intestinal tissues (ileum) were fixed and stained with H\&E. Representative images are shown. Histologic scores were evaluated using H\&E slides. (A) H\&E staining of small intestine (ileum); (B) inflammation scores; (C) villus lengths; (D) crypt lengths ( $n=5-13$ mice per group). Scatter plot: horizontal bar, median. S, sham; E, ethanol binge; M, SkMSCs. Significance between treated groups was determined using Mann-Whitney $U$ test. $* P<0.05$, $* * * ~ P<0.001 ;$ ns, no statistical significance.

\section{Effect of SkMSCs on intestinal histopathology in binge ethanol-treated mice}

Binge ethanol-induced hepatic injury is mediated by intestinal inflammation [27, 28]. Moreover, binge ethanol-induced damage to the small intestine is characterized by changes in the length of the villi and crypts $[35,36]$. As SkMSCs decreased the translocation of LPS, bacteria, and inflammatory markers in the liver, it was further hypothesized that these cells could decrease ethanol-induced damage to the intestinal tissue (ileum). To test this hypothesis, tissue damage was assessed in the ileum via H\&E staining. Ethanol-exposed mice administered SkMSCs showed reduced infiltration of inflammatory cells with diminished lengths of villi and crypt compared to mice administered binge ethanol alone (Figure 4A-D). No significant changes in inflammation scores and lengths of villi and crypts were observed between the sham and SkMSC-treated groups (Figure 4A-D), suggesting that treatment with SkMSCs does not affect intestinal histology. These findings suggest that SkMSCs decrease ethanol-induced hepatic injury by suppressing intestinal inflammation.

\section{Effects of SkMSCs on blood albumin leakage and inflammatory mediators in the liver of binge ethanol-treated mice}

Inflammation of the small intestine induced by ethanol binge drinking disrupts gut permeability, which induces the leakage of albumin into the intestinal lumen from blood [28, 37, 38]. As SkMSCs decreased binge ethanol-induced tissue damage in the small intestine based on histological assessments (Figure 4A-D), we proceeded to determine whether treatment with SkMSCs could diminish the expression of inflammatory cytokines in the liver using qRT-PCR. Ethanol-exposed mice administered 
SkMSCs showed reduced expression levels of $I L-1 \beta$, TNF- $\alpha$, and iNOS compared to those in mice administered ethanol alone (Figure 5A-C). Additionally, to test the barrier integrity of the small intestine, albumin was further examined in stool samples via ELISA. Binge ethanol-treated mice administered SkMSCs showed decreased levels of albumin compared to mice treated with ethanol alone (Figure 5D). Such finding suggests that SkMSCs decrease binge ethanol-induced liver inflammation by inhibiting intestinal inflammation.

A

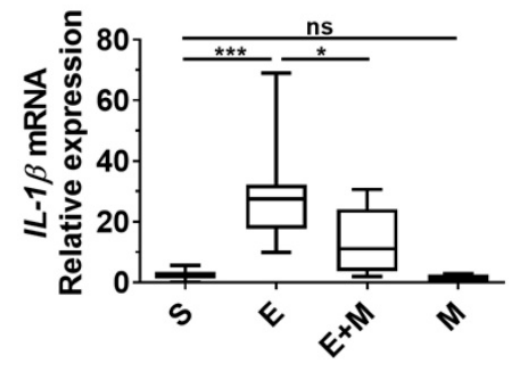

C

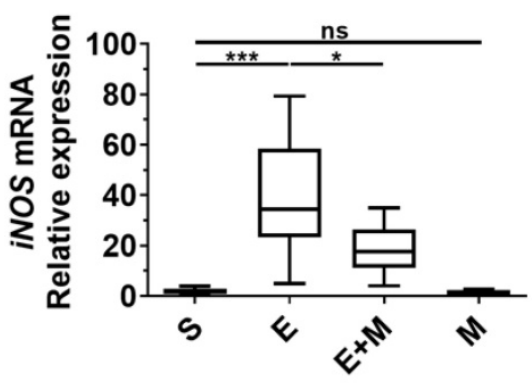

Effects of HGF on the intestinal epithelial cells, Caco-2/tc7

We sought to determine how SkMSC administration reduced alcohol-induced liver injury. Previously, we demonstrated that SkMSCs secrete more hepatocyte growth factor (HGF) than adipose tissue-derived mesenchymal stem cells (ASCs) [26]. Before investigating the HGF-induced protective mechanisms, we checked the basal level of HGF in the culture supernatants of SkMSCs. As expected, SkMSCs significantly produced HGF (Figure 6A). As

B

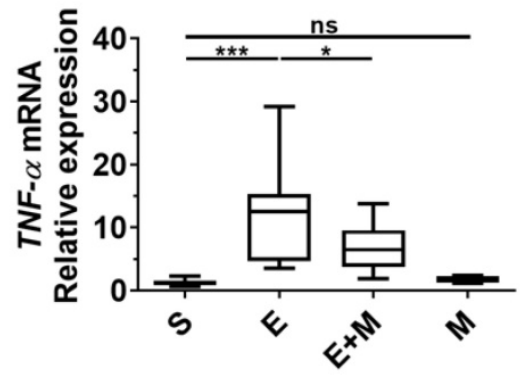

D

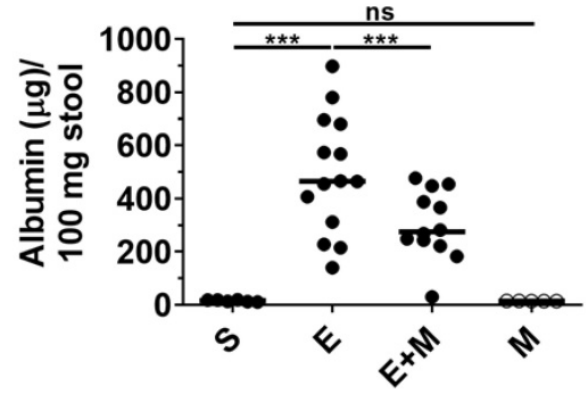

Figure 5. Effect of skeletal muscle satellite cell-derived mesenchymal stem cells (SkMSCs) on pro-inflammatory genes in the small intestine (ileum) and albumin levels in stool in binge ethanol-treated mice. Eight-week-old C57BL/6 female mice were treated with binge ethanol. Mice were sacrificed on day 1 , and liver tissues were analyzed to determine IL-I $\beta$, TNF- $\alpha$, and iNOS expression levels using qRT-PCR. The stool of mice was analyzed to detect albumin leakage by ELISA. (A) IL-I $\beta$ expression; (B) TNF- $\alpha$ expression; (C) iNOS expression; (D) albumin levels in stool. ( $n=5-12$ mice per group). Scatter plot: horizontal bar, median. S, sham; E, ethanol binge; M, SkMSCs. Significance between treated groups was determined using Mann-Whitney $U$ test. $* P<0.05$, $* * * P<0.001$; ns, no statistical significance.

A

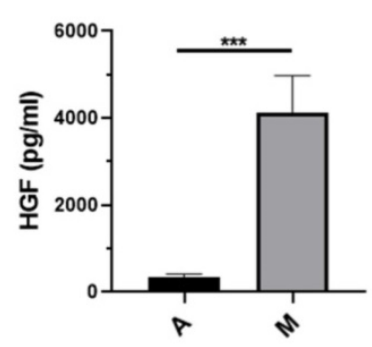

B

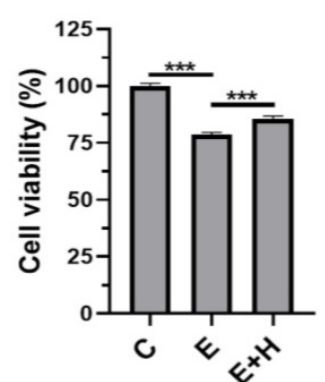

C

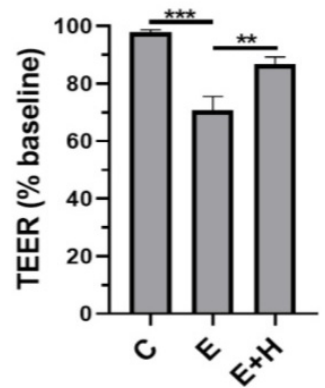

D

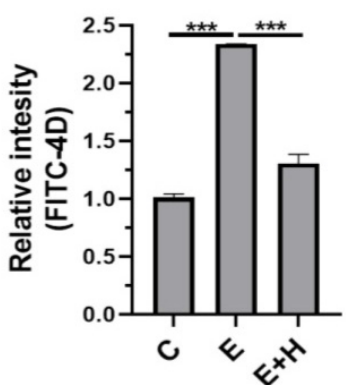

Figure 6. Treatment with HGF restores cell viability and permeability in ethanol-exposed intestinal epithelial cells. Human ASCs and SkMSCs were monocultured for $48 \mathrm{~h}$. Thereafter, HGF applied to the culture supernatants of ASCs or SkMSCs was assessed via ELISA. Human intestinal Caco-2/tc7 cells were treated with HGF for $24 \mathrm{~h}$. Cell viability was measured via the MTT assay. The barrier function of Caco-2/tc7 cells was measured for transepithelial electrical resistance (TEER) and translocated FITC-4k dextran (A) Secreted HGF in culture supernatants of ASCs or SkMSCs. (B) Cell viability of ethanol-exposed Caco-2/tc7 cells treated with HGF (10 ng/ml). (C) TEER of Caco-2/tc7 cells treated with HGF for 24 h. (D) Relative intensities of FITC-4k dextran in transwell seeded with Caco-2/tc7 monolayers treated with HGF for 24 h. A, ASCs; M, SkMSCs; C, control; E, ethanol; $\mathrm{H}$, hepatocyte growth factor. Significance between treated groups was determined using Mann-Whitney $U$ test. $* * P<0.01$, $* * * P<0.001 ;$ ns, no statistical significance. 
binge alcohol-induced liver injury is mediated by disruption of the epithelial barrier, we hypothesized that HGF directly protects against ethanol-induced damage to the intestinal barrier. To test this hypothesis, the human intestinal epithelial cell line, Caco-2/tc7 cells, was co-treated with $40 \mathrm{mM}$ ethanol and HGF $(20 \mathrm{ng} / \mathrm{mL})$ for $24 \mathrm{~h}$. Thereafter, viability assay (MTT assay), permeability test (trans-epithelial electrical resistance [TEER] analysis, and FITC-4k dextran assay [FITC-D4] were conducted. Ethanolexposed Caco-2/tc7 cells displayed decreased cell viability compared to control cells (Figure 6B). Further, Caco-2/tc7 cells co-treated with $40 \mathrm{mM}$ ethanol and HGF $(20 \mathrm{ng} / \mathrm{mL})$ for $24 \mathrm{~h}$ showed increased cell viability compared with $\mathrm{Caco}-2 / \mathrm{tc} 7$ cells treated with $40 \mathrm{mM}$ ethanol alone (Figure 6C). Consistently, treatment with HGF recovered the decrease in TEER and increased the permeation of FITC-D4 (Figure 6C, D). These results indicate that the protective roles of SkMSCs in ethanol-induced liver injury and gut leakiness are mediated by HGFinduced protection of intestinal epithelial cells against ethanol-induced damage.

\section{Discussion}

To our knowledge, the present study is the first to demonstrate the anti-inflammatory effects of SkMSCs on ethanol-induced hepatic and intestinal inflammation. Histological analysis revealed decreased extent of inflammation in the small intestines of SkMSC-treated ethanol-fed mice compared to that in binge ethanol-treated mice. Moreover, inflammation-associated cytokine levels were reduced in the livers and intestines of SkMSC-treated ethanol-fed mice. We hypothesize that the SkMSC-induced suppression of hepatic injury in the binge ethanol model was mediated by diminishing intestinal inflammation.

Intestinal inflammation induced by ethanol or fructose involves the destruction of the intestinal barrier, which in turn leads to gut leakiness [27, 28, 34]. Gut leakage induced by ethanol binding in vivo may be directly caused by ethanol itself. For example, ethanol $(80 \mathrm{mM})$ treatment induces the downregulation of barrier function by increasing the levels of CYP2E1/reactive oxygen species- or iNOS/reactive nitrogen species-mediated protein degradation of tight junctions in T84 or Caco-2 intestinal epithelial cells [28, 39-41]. As FAPs can migrate to inflamed tissues enriched with CXCL via the CXCR receptor $[42,43]$, their migration might be specifically effective in the targeting of intestinal epithelial cells in the inflamed gut by downregulating the expression of CYP2E1 or iNOS in a paracrine manner.
The compromised integrity of the gut barrier is characterized by increased levels of fluorescein isothiocyanate-dextran, LPS, and fecal albumin, which are indications of increased intestinal permeability based on several animal studies [44-46]. Diet-related animal studies have revealed the critical role of the intestinal barrier in preventing liver inflammation due to the translocation of bacterial products or gut bacteria $[34,47,48]$. However, we did not investigate permeability with fluorescein isothiocyanate-dextran in a binge ethanol model after the administration of SkMSCs in vivo. However, we revealed elevated levels of LPS and albumin in the serum and stool (Figure 3A, B, and Figure 5D). Further, HGF treatment was found to rescue ethanol-induced barrier disruption in vitro (Figure 6). HGF might inhibit CYP2E1-mediated nitro-oxidative stress by ethanol in intestinal epithelial cells, thereby suppressing the degradation of junctional proteins. Further studies are required to understand the mechanism of SkMSC/HGF in detail.

Hepatic inflammation and steatosis (an accumulation of lipid droplets) are promoted by the translocation of LPS from gram-negative bacteria in several models of fatty liver diseases [49-51]. LPS is a ligand for TLR4 receptors on innate immune cells, including neutrophils and macrophages $[52,53]$, and induces IL-1 $\beta$, TNF- $\alpha$, reactive oxygen species, and reactive nitrogen species, which trigger hepatic injury and steatosis [54, 55]. In our study, we did not investigate infiltrated neutrophils or macrophages in the hepatic tissues of mice treated with binge ethanol or SkMSCs. However, treatment with SkMSCs may directly decrease the activation of LPS-activated neutrophils or macrophages. Additional studies are required to discern the SkMSC-mediated mechanism underlying the suppression of ethanol-induced liver injury in terms of lipogenesis.

Previously, we performed an analysis of the transcriptome of SkMSCs using next generation sequencing and revealed that the expression levels of COX-2 and HGF were significantly increased in SkMSCs co-cultured with IFN- $\gamma-$ and LPS-treated THP-1 macrophages [26]. COX-2 leads to an increase in PGE2 level $[56,57]$ for the mediation of an anti-inflammatory neutrophil phenotype at sites of tissue injury in vivo [58]. PGE2 has also been reported to induce M2 polarization of macrophages in inflammatory tissues $[59,60]$. Secreted PGE2 or HGF has also been found to suppress the inflammatory status of M1 macrophages in vitro and in vivo [61, 62]. Specifically, Choi et al. showed that HGF level increases the transition from M1 macrophages to the M2 phenotype through activation of AMPK signaling and a decrease in the M1 phenotype-associated 
inflammatory cytokines (IL-1 $\beta$, TNF- $\alpha$, and iNOS) in an LPS-treated murine macrophage cell line [61]. Herein, we did not investigate AMPK-related signaling in macrophages. PGE2 or HGF by SkMSCs might decrease ethanol-induced hepatic injury by suppressing inflammatory M1 macrophages or promoting anti-inflammatory M2 macrophages; this is because alcoholic liver diseases are exacerbated by inflammatory neutrophils and macrophages. In addition to the anti-inflammatory effects of PGE2 and HGF, PGE2 and HGF have been reported to promote the regeneration of intestinal epithelial cells [63, 64]. In a previous study, SkMSCs co-cultured with macrophages produced both PGE2 and HGF; however, SkMSCs cultured alone expressed only HGF at high levels. Therefore, in this study, the effect of HGF on the intestinal epithelial cells, Caco-2/tc7, was analyzed in vitro. Based on our findings, HGF protects intestinal epithelial cells from alcohol-induced damage. As PGE2 can also act on the regeneration of intestinal epithelial cells, both PGE2 and HGF produced by SkMSCs may reduce intestinal inflammatory responses and have protective effects on intestinal epithelial cells in acute alcohol-induced liver injury. As the direct mechanism of HGF and PGE2 production by SkMSCs has not been validated in a model of acute alcohol-induced liver injury, our study had limitations.

Taken together, our study provides evidence that SkMSCs can be used to inhibit ethanol-induced liver injury, potentially by reducing levels of intestinal inflammation. Although the ability of SkMSCs to diminish tissue inflammation is expected, our study unveils the potential strategy of targeting intestinal inflammation to prevent ethanol-induced liver damage. Future studies would be critical to evaluate the therapeutic efficacy of SkMSCs in gut-mediated chronic inflammatory diseases in vivo.

\section{Conclusions}

This study revealed the anti-inflammatory effects of human SkMSCs in an ethanol-induced liver injury model. Treating human skeletal tissue with SkMSCs suppressed hepatic inflammation and injury by reducing gut inflammation and leakiness caused by excessive ethanol intake. These findings may prove useful to assess the outcomes of future SkMSC-based therapies in humans with alcoholic liver diseases.

\section{Acknowledgements}

This research was funded by the Basic Science Research Program through the National Research Foundation of Korea (2017R1D1A1A02019212 and 2017R1D1A1A02018088) and NRF-2017-Fostering Core Leaders of the Future Basic Science
Program/Global Ph.D. Fellowship Program (2017H1A2A1045727).

\section{Competing Interests}

The authors have declared that no competing interest exists.

\section{References}

1. Tapper EB, Parikh ND. Mortality due to cirrhosis and liver cancer in the United States, 1999-2016: observational study. BMJ. 2018; 362: k2817.

2. Ventura-Cots $M$, Watts $A E$, Bataller $R$. Binge drinking as a risk factor for advanced alcoholic liver disease. Liver Int. 2017; 37: 1281-3.

3. Zakhari S, Li TK. Determinants of alcohol use and abuse: Impact of quantity and frequency patterns on liver disease. Hepatology. 2007; 46: 2032-9.

4. Bala S, Marcos M, Gattu A, Catalano D, Szabo G. Acute binge drinking increases serum endotoxin and bacterial DNA levels in healthy individuals. PLoS One. 2014; 9: e96864.

5. Bjarnason I, Peters TJ, Wise RJ. The leaky gut of alcoholism: possible route of entry for toxic compounds. Lancet. 1984; 1: 179-82.

6. Orio L, Anton M, Rodriguez-Rojo IC, Correas A, Garcia-Bueno B, Corral M, et al. Young alcohol binge drinkers have elevated blood endotoxin, peripheral inflammation and low cortisol levels: neuropsychological correlations in women. Addict Biol. 2018; 23: 1130-44.

7. Blednov YA, Black M, Chernis I, Da Costa A, Mayfield J, Harris RA. Ethanol Consumption in Mice Lacking CD14, TLR2, TLR4, or MyD88. Alcohol Clin Exp Res. 2017; 41: 516-30

8. Jia L, Chang X, Qian S, Liu C, Lord CC, Ahmed N, et al. Hepatocyte toll-like receptor 4 deficiency protects against alcohol-induced fatty liver disease. Mol Metab. 2018; 14: 121-9.

9. Karoly HC, Ellingson JM, Hutchison KE. Interactions between TLR4 methylation and alcohol consumption on subjective responses to an alcohol infusion. Alcohol Alcohol. 2018; 53: 650-8.

10. Adachi Y, Moore LE, Bradford BU, Gao W, Thurman RG. Antibiotics prevent liver injury in rats following long-term exposure to ethanol. Gastroenterology. 1995; 108: 218-24.

11. Le Roy T, Llopis M, Lepage P, Bruneau A, Rabot S, Bevilacqua C, et al. Intestinal microbiota determines development of non-alcoholic fatty liver disease in mice. Gut. 2013; 62: 1787-94.

12. M CCC, N LL, C MF, J LG, D A, C G, et al. Comparing the effects of acute alcohol consumption in germ-free and conventional mice: the role of the gut microbiota. BMC Microbiol. 2014; 14: 240.

13. European Association for the Study of the Liver. Electronic address eee. EASL Clinical Practice Guidelines: Liver transplantation. J Hepatol. 2016; 64: 433-85.

14. Jang YO, Kim MY, Cho MY, Baik SK, Cho YZ, Kwon SO. Effect of bone marrow-derived mesenchymal stem cells on hepatic fibrosis in a thioacetamide-induced cirrhotic rat model. BMC Gastroenterol. 2014; 14: 198.

15. Kharaziha P, Hellstrom PM, Noorinayer B, Farzaneh F, Aghajani K, Jafari F, et al. Improvement of liver function in liver cirrhosis patients after autologous mesenchymal stem cell injection: a phase I-II clinical trial. Eur J Gastroenterol Hepatol. 2009; 21: 1199-205.

16. Wu CX, Wang D, Cai Y, Luo AR, Sun H. Effect of Autologous Bone Marrow Stem Cell Therapy in Patients with Liver Cirrhosis: A Meta-analysis. J Clin Transl Hepatol. 2019; 7: 238-48.

17. $\mathrm{Wu} \mathrm{DB}, \mathrm{Chen} \mathrm{EQ}$, Tang $\mathrm{H}$. Stem cell transplantation for the treatment of end-stage liver disease. World J Hepatol. 2018; 10: 907-10.

18. Huang S, Xu L, Sun Y, Wu T, Wang K, Li G. An improved protocol for isolation and culture of mesenchymal stem cells from mouse bone marrow. J Orthop Translat. 2015; 3: 26-33.

19. Hass R, Kasper C, Bohm S, Jacobs R. Different populations and sources of human mesenchymal stem cells (MSC): A comparison of adult and neonatal tissue-derived MSC. Cell Commun Signal. 2011; 9: 12.

20. Wang $Y$, Chen $X$, Cao W, Shi Y. Plasticity of mesenchymal stem cells in immunomodulation: pathological and therapeutic implications. Nat Immunol. 2014; 15: 1009-16.

21. Gnecchi M, Danieli P, Malpasso G, Ciuffreda MC. Paracrine Mechanisms of Mesenchymal Stem Cells in Tissue Repair. Methods Mol Biol. 2016; 1416: 123-46.

22. Biferali B, Proietti D, Mozzetta C, Madaro L. Fibro-Adipogenic Progenitors Cross-Talk in Skeletal Muscle: The Social Network. Front Physiol. 2019; 10: 1074

23. Low M, Eisner C, Rossi F. Fibro/Adipogenic Progenitors (FAPs): Isolation by FACS and Culture. Methods Mol Biol. 2017; 1556: 179-89.

24. Malecova B, Gatto S, Etxaniz U, Passafaro M, Cortez A, Nicoletti C, et al. Dynamics of cellular states of fibro-adipogenic progenitors during myogenesis and muscular dystrophy. Nat Commun. 2018; 9: 3670.

25. Boonen KJ, Post MJ. The muscle stem cell niche: regulation of satellite cells during regeneration. Tissue Eng Part B Rev. 2008; 14: 419-31.

26. Kim S, Jung PY, Lee JS, Hwang S, Sohn JH, Yoon Y, et al. Cultured human skeletal muscle satellite cells exhibit characteristics of mesenchymal stem cells 
and play anti-inflammatory roles through prostaglandin E2 and hepatocyte growth factors. Cell Biol Int. 2021.

27. Cho YE, Song BJ. Pomegranate prevents binge alcohol-induced gut leakiness and hepatic inflammation by suppressing oxidative and nitrative stress. Redox Biol. 2018; 18: 266-78.

28. Cho YE, Yu LR, Abdelmegeed MA, Yoo SH, Song BJ. Apoptosis of enterocytes and nitration of junctional complex proteins promote alcohol-induced gut leakiness and liver injury. J Hepatol. 2018; 69: 142-53.

29. Abdelmegeed MA, Banerjee A, Jang S, Yoo SH, Yun JW, Gonzalez FJ, et al. CYP2E1 potentiates binge alcohol-induced gut leakiness, steatohepatitis, and apoptosis. Free Radic Biol Med. 2013; 65: 1238-45.

30. Carmiel-Haggai M, Cederbaum AI, Nieto N. Binge ethanol exposure increases liver injury in obese rats. Gastroenterology. 2003; 125: 1818-33.

31. Muller AF, Toghill PJ. Functional hyposplenism in alcoholic liver disease: a toxic effect of alcohol? Gut. 1994; 35: 679-82.

32. Kirkineska L, Perifanis V, Vasiliadis T. Functional hyposplenism. Hippokratia. 2014; 18: 7-11

33. Corazza GR, Addolorato G, Biagi F, Caputo F, Castelli E, Stefanini GF, et al. Splenic function and alcohol addiction. Alcohol Clin Exp Res. 1997; 21: 197-200.

34. Cho YE, Kim DK, Seo W, Gao B, Yoo SH, Song BJ. Fructose Promotes Leaky Gut, Endotoxemia, and Liver Fibrosis Through Ethanol-Inducible Cytochrome P450-2E1-Mediated Oxidative and Nitrative Stress. Hepatology. 2021; 73: 2180-2195.

35. Barona E, Pirola RC, Leiber CS. Small intestinal damage and changes in cell population produced by ethanol ingestion in the rat. Gastroenterology. 1974; 66: 226-34.

36. Kirpich IA, Feng W, Wang Y, Liu Y, Beier JI, Arteel GE, et al. Ethanol and dietary unsaturated fat (corn oil/linoleic acid enriched) cause intestinal inflammation and impaired intestinal barrier defense in mice chronically fed alcohol. Alcohol. 2013; 47: 257-64.

37. Chen P, Starkel P, Turner JR, Ho SB, Schnabl B. Dysbiosis-induced intestinal inflammation activates tumor necrosis factor receptor I and mediates alcoholic liver disease in mice. Hepatology. 2015; 61: 883-94.

38. Wang L, Fouts DE, Starkel P, Hartmann P, Chen P, Llorente C, et al. Intestinal REG3 Lectins Protect against Alcoholic Steatohepatitis by Reducing Mucosa-Associated Microbiota and Preventing Bacterial Translocation. Cell Host Microbe. 2016; 19: 227-39.

39. Elamin E, Masclee A, Dekker J, Jonkers D. Ethanol disrupts intestinal epithelial tight junction integrity through intracellular calcium-mediated Rho/ROCK activation. Am J Physiol Gastrointest Liver Physiol. 2014; 306: G677-85.

40. Forsyth CB, Voigt RM, Shaikh M, Tang Y, Cederbaum AI, Turek FW, et al. Role for intestinal CYP2E1 in alcohol-induced circadian gene-mediated intestinal hyperpermeability. Am J Physiol Gastrointest Liver Physiol. 2013; 305: G185-95.

41. Wang Y, Tong J, Chang B, Wang B, Zhang D, Wang B. Effects of alcohol on intestinal epithelial barrier permeability and expression of tight junction-associated proteins. Mol Med Rep. 2014; 9: 2352-6.

42. Marinkovic M, Fuoco C, Sacco F, Cerquone Perpetuini A, Giuliani G, Micarelli E, et al. Fibro-adipogenic progenitors of dystrophic mice are insensitive to NOTCH regulation of adipogenesis. Life Sci Alliance. 2019; 2.

43. Oprescu SN, Yue F, Qiu J, Brito LF, Kuang S. Temporal Dynamics and Heterogeneity of Cell Populations during Skeletal Muscle Regeneration. iScience. 2020; 23: 100993.

44. Han $\mathrm{Y}$, Zhao $\mathrm{T}$, Cheng $\mathrm{X}$, Zhao $\mathrm{M}$, Gong $\mathrm{SH}$, Zhao $\mathrm{YQ}$, et al. Cortical Inflammation is Increased in a DSS-Induced Colitis Mouse Model. Neurosci Bull. 2018; 34: 1058-66.

45. Thim-Uam A, Surawut S, Issara-Amphorn J, Jaroonwitchawan T, Hiengrach $P$ Chatthanathon $\mathrm{P}$, et al. Leaky-gut enhanced lupus progression in the $\mathrm{FC}$ gamma receptor-IIb deficient and pristane-induced mouse models of lupus. Sci Rep. 2020; 10: 777.

46. Yoshino $\mathrm{S}$, Sasatomi $\mathrm{E}$, Mori $\mathrm{Y}$, Sagai M. Oral administration of lipopolysaccharide exacerbates collagen-induced arthritis in mice. J Immunol. 1999; 163: 3417-22.

47. Guerville M, Leroy A, Sinquin A, Laugerette F, Michalski MC, Boudry G. Western-diet consumption induces alteration of barrier function mechanisms in the ileum that correlates with metabolic endotoxemia in rats. Am J Physiol Endocrinol Metab. 2017; 313: E107-E20.

48. Kim KA, Gu W, Lee IA, Joh EH, Kim DH. High fat diet-induced gut microbiota exacerbates inflammation and obesity in mice via the TLR4 signaling pathway. PLoS One. 2012; 7: e47713.

49. Fukunishi S, Sujishi $T$, Takeshita A, Ohama $H$, Tsuchimoto $Y$, Asai A, et al. Lipopolysaccharides accelerate hepatic steatosis in the development of nonalcoholic fatty liver disease in Zucker rats. J Clin Biochem Nutr. 2014; 54: $39-44$.

50. Guo H, Diao N, Yuan R, Chen K, Geng S, Li M, et al. Subclinical-Dose Endotoxin Sustains Low-Grade Inflammation and Exacerbates Steatohepatitis in High-Fat Diet-Fed Mice. J Immunol. 2016; 196: 2300-8.

51. Yang L, Miura $K$, Zhang B, Matsushita $H$, Yang YM, Liang S, et al. TRIF Differentially Regulates Hepatic Steatosis and Inflammation/Fibrosis in Mice. Cell Mol Gastroenterol Hepatol. 2017; 3: 469-83.

52. O'Mahony DS, Pham U, Iyer R, Hawn TR, Liles WC. Differential constitutive and cytokine-modulated expression of human Toll-like receptors in primary neutrophils, monocytes, and macrophages. Int J Med Sci. 2008; 5: 1-8.
53. Park BS, Lee JO. Recognition of lipopolysaccharide pattern by TLR4 complexes. Exp Mol Med. 2013; 45: e66.

54. Diehl AM, Li ZP, Lin HZ, Yang SQ. Cytokines and the pathogenesis of non-alcoholic steatohepatitis. Gut. 2005; 54: 303-6.

55. Torer N, Ozenirler S, Yucel A, Bukan N, Erdem O. Importance of cytokines, oxidative stress and expression of BCL-2 in the pathogenesis of non-alcoholic steatohepatitis. Scand J Gastroenterol. 2007; 42: 1095-101.

56. Chen EP, Smyth EM. COX-2 and PGE2-dependent immunomodulation in breast cancer. Prostaglandins Other Lipid Mediat. 2011; 96: 14-20.

57. Muraoka N, Nara K, Tamura F, Kojima H, Yamakawa H, Sadahiro T, et al. Role of cyclooxygenase-2-mediated prostaglandin E2-prostaglandin E receptor 4 signaling in cardiac reprogramming. Nat Commun. 2019; 10: 674

58. Loynes CA, Lee JA, Robertson AL, Steel MJ, Ellett F, Feng Y, et al. PGE2 production at sites of tissue injury promotes an anti-inflammatory neutrophil phenotype and determines the outcome of inflammation resolution in vivo. Sci Adv. 2018; 4: eaar8320.

59. Luan B, Yoon YS, Le Lay J, Kaestner KH, Hedrick S, Montminy M. CREB pathway links PGE2 signaling with macrophage polarization. Proc Natl Acad Sci U S A. 2015; 112: 15642-7.

60. Sheppe AEF, Kummari E, Walker A, Richards A, Hui WW, Lee JH, et al. PGE2 Augments Inflammasome Activation and M1 Polarization in Macrophages Infected With Salmonella Typhimurium and Yersinia enterocolitica. Front Microbiol. 2018; 9: 2447.

61. Choi W, Lee J, Lee J, Lee SH, Kim S. Hepatocyte Growth Factor Regulates Macrophage Transition to the M2 Phenotype and Promotes Murine Skeletal Muscle Regeneration. Front Physiol. 2019; 10: 914.

62. Park HJ, Kim J, Saima FT, Rhee KJ, Hwang S, Kim MY, et al. Adipose-derived stem cells ameliorate colitis by suppression of inflammasome formation and regulation of M1-macrophage population through prostaglandin E2. Biochem Biophys Res Commun. 2018; 498: 988-95.

63. Kanayama M, Takahara $\mathrm{T}$, Yata $\mathrm{Y}$, Xue $\mathrm{F}$, Shinno $\mathrm{E}$, Nonome $\mathrm{K}$, et al Hepatocyte growth factor promotes colonic epithelial regeneration via Akt signaling. Am J Physiol Gastrointest Liver Physiol. 2007; 293: G230-9.

64. Miyoshi H, VanDussen KL, Malvin NP, Ryu SH, Wang Y, Sonnek NM, et al. Prostaglandin E2 promotes intestinal repair through an adaptive cellular response of the epithelium. EMBO J. 2017; 36: 5-24. 